\title{
Contribuição da medicina nuclear para a avaliação dos linfomas
}

\author{
Marcelo T. Sapienza $a^{1,2}$ \\ Marília M. S. Marone ${ }^{1,3}$ \\ Carlos S. Chiattone ${ }^{3}$
}

\begin{abstract}
A medicina nuclear permite a caracterização funcional de tecidos, acrescentando dados à avaliação anatômica realizada por outros métodos de imagem, como a tomografia computadorizada, ressonância magnética ou ultrasom. Além do diagnóstico e estadiamento, as informações funcionais fornecidas pela medicina nuclear são particularmente úteis no seguimento e avaliação de resposta terapêutica dos pacientes com linfoma. A presença de massa residual após quimioterapia ou a infiltração de linfonodos de pequenas dimensões são exemplos de situações nas quais a avaliação isolada de características anatômicas é insuficiente. Os principais métodos utilizados são a cintilografia com gálio67, traçador com alta afinidade por transferrina e receptores de ferro, e os estudos com flúor-deoxiglicose, emissor de pósitron que permite a caracterização do metabolismo de glicose nos tecidos. Outros traçadores, como o tálio-201, sestamibi-Tc99m e octreotide-In111, também são utilizados em menor escala na avaliação dos pacientes com linfoma. Os mecanismos de captação, principais indicações dos estudos, limitações e resultados de literatura serão revistos. Rev.bras.hematol.hemoter., 2001, 23(2): 79-92
\end{abstract}

Palavras-chave: Linfoma, cintilografia, gálio-67, PET

\section{Introdução}

Os métodos de imagem têm importante papel na avaliação não invasiva dos pacientes com linfoma. A tomografia computadorizada (TC), a ressonância magnética (RM) e o ultrasom permitem caracterizar alterações anatômicas decorrentes da doença, porém a avaliação anatômica nem sempre é conclusiva. A presença de massa residual em pacientes com boa resposta ao tratamento, assim como a infiltração de linfonodos de pequenas dimensões, são exemplos de situações nas quais a avaliação estrutural é insuficiente.
Ao permitir a caracterização funcional e metabólica dos tecidos, a medicina nuclear complementa os dados anatômicos e, desta forma, auxilia no diagnóstico e principalmente no acompanhamento e otimização do tratamento dos pacientes com linfoma. Serão a seguir abordados os principais métodos empregados, com destaque para a cintilografia com gálio-67 e O PET com ${ }^{18} \mathrm{~F}$ fluordeoxiglucose, método com perspectivas crescentes de utilização em nosso meio devido a instalação de equipamentos e produção local do radiofármaco.

\footnotetext{
1 - UDDO - Serviço de Medicina Nuclear do Hospital Samaritano de São Paulo

2 - Centro de Medicina Nuclear da Faculdade de Medicina da Universidade de São Paulo

3 - Faculdade de Ciências Médicas da Santa Casa de Misericórdia de São Paulo
}

Correspondência: Marcelo Tatit Sapienza

Rua Barão de Melgaço, 230 apto 24. 05684-030. São Paulo. SP

Fone/fax: (11) 3825-4433

E-mail: tatit@attglobal.net 


\section{Cintilografia com Gálio-670}

\section{Aspectos gerais}

O uso do gálio-67 para detecção de linfomas foi descrito há mais de 30 anos (1), tendo havido considerável melhoria nas técnicas empregadas durante este período, com otimização das doses e introdução das técnicas tomográficas, bem como uma melhor definição de suas aplicações.

O gálio-67 é um isótopo radioativo com características análogas ao ferro, ligando-se à transferrina plasmática após sua administração intravenosa. Além de tumores e processos inflamatórios, o gálio-67 é normalmente concentrado pelo fígado, baço e medula óssea, sendo eliminado por via urinária (predominante nas primeiras 24 horas) e gastrointestinal. Dentre os fatores locais que levam a um maior aporte de gálio-67 aos tumores, destacam-se o aumento de fluxo e permeabilidade vascular. A captação e fixação do isótopo a nível celular ocorre principalmente pela ligação do complexo gáliotransferrina a receptores de membrana, seguida de transporte para os lisossomas (2). É demonstrado que a variação de captação de gálio-67 nos diferentes subtipos de linfoma nãoHodgkin (LNH), com maior captação nos linfomas de alto grau, é diretamente relacionada à expressão de receptores de transferrina na membrana celular (3).

Além da variação do grau de captação pelos diferentes subtipos histológicos, relata-se também redução de sensibilidade para lesões com dimensões abaixo de $2 \mathrm{~cm}$ (pela resolução do método) ou acima de $5 \mathrm{~cm}$ (devido a necrose). A localização das lesões é outro fator importante de variação da sensibilidade, a ser abordado adiante. A captação tumoral do gálio-67 pode ser reduzida por radio e quimioterapia recentes, em especial quando empregadas cisplatina, bleomicina ou vincristina, recomendando-se um intervalo superior a 3 semanas para o estudo. Este intervalo é sugerido mesmo para pacientes estudados durante o tratamento quimioterápico (ou seja, a cintilografia deve ser feita imediatamente antes do próximo ciclo) (4). Outros fatores que levam à modificação da biodistribuição do gálio-67, com possível queda da sensibilidade, são o aumento do pool de ferro ou transfusões sangüíneas. Os contrastes paramagnéticos usados em ressonância magnética também podem levar à redução da captação hepato-esplênica e aumento da captação óssea por até 96 horas (5).

Dentre as causas de estudos falso positivos destacam-se as patologias inflamatórias como a tuberculose, blastomicose e sarcoidose. A captação em projeção hilar, geralmente discreta e simétrica, pode ser observada em idosos, associada a bronquite ou tabagismo (6). A hiperplasia do timo (que normalmente capta o gálio-67) é outra causa de estudos falso positivos nos pacientes com idade inferior a 25 anos. É observada em até 17\% das crianças e ocorre de forma transitória durante ou até 4 a 10 meses após o término da quimioterapia $(7,8,9)$.

A cintilografia habitualmente é feita pela aquisição de imagens seriadas, entre 1 a 3 dias após a administração de 3 a 5 mCi de gálio-67. Alguns autores têm sugerido a utilização de atividades mais altas do radioisótopo (8-10 mCi), permitindo o emprego mais amplo das técnicas tomográficas (SPECT), bem como a realização de imagens com intervalos de até 7 a 10 dias após a injeção $(10,11,12)$. Estas técnicas aumentam a acurácia principalmente na detecção de doença abdominal, prejudicada pela distribuição fisiológica do traçador.

\section{Avaliação inicial e estadiamento}

A primeira descrição de utilização do gálio-67 na detecção de tumores foi feita por Edward e Hayes em 1969 (1). Estudos mais recentes mostram sensibilidade de 86 a $97 \%$ do gálio-67 no diagnóstico inicial e estadiamento de pacientes com doença de Hodgkin (DH) não tratados e de 86 a 92\% para linfomas não-Hodgkin (LNH), com alta especificidade (13). Em relação a localização da lesão, a sensibilidade dos estudos tomográficos (SPECT) é próxima a 96\% para tórax, $60 \%$ para abdome e $83 \%$ para as lesões periféricas (14, 15). Tumeh, em 1987, demonstrou o aumento de sensibilidade e especificidade da técnica tomográfica tanto para lesões abdominais (imagens planas: sens. 69\% esp. 87\%, SPECT: sens. 85\% esp. 100\%) como torácicas (imagens planas: sens. e esp. 66\%, SPECT: sens. 96\% esp. 100\%) (16). Outros 
autores, entretanto, referem valores mais baixos de sensibilidade, próxima a $80 \%$ para $\mathrm{DH}$ e de 60 a 70\% para LNH (4). Hagemeister descreve sensibilidade de apenas 64\% no estadiamento inicial de 32 pacientes com DH e detecção de apenas 5\% das lesões em estágio pré-clínico em outros 20 pacientes (17).

Apesar da variação de sensibilidade na literatura, é um consenso que os métodos anatômicos, como a TC e RM, são os mais indicados para estadiamento inicial. O gálio-67 pode ocasionalmente modificar o estádio, pela detecção de acometimento extra-nodal ou torácico. O SPECT com gálio-67 aumentou o estádio frente aos métodos convencionais em 3 de 44 pacientes (7\%), estudados antes do tratamento, principalmente pela detecção de doença torácica. Em 16\% dos pacientes o gálio não foi capaz de detectar lesões infradiafragmáticas (18).

Assim como a localização das lesões, o tipo histológico do linfoma é outro fator determinante da sensibilidade, maior para $\mathrm{DH}$ e LNH de grau intermediário/alto $(10,19)$, sendo discutível se esta limitação, decorrente da menor afinidade celular, pode ser melhorada com o

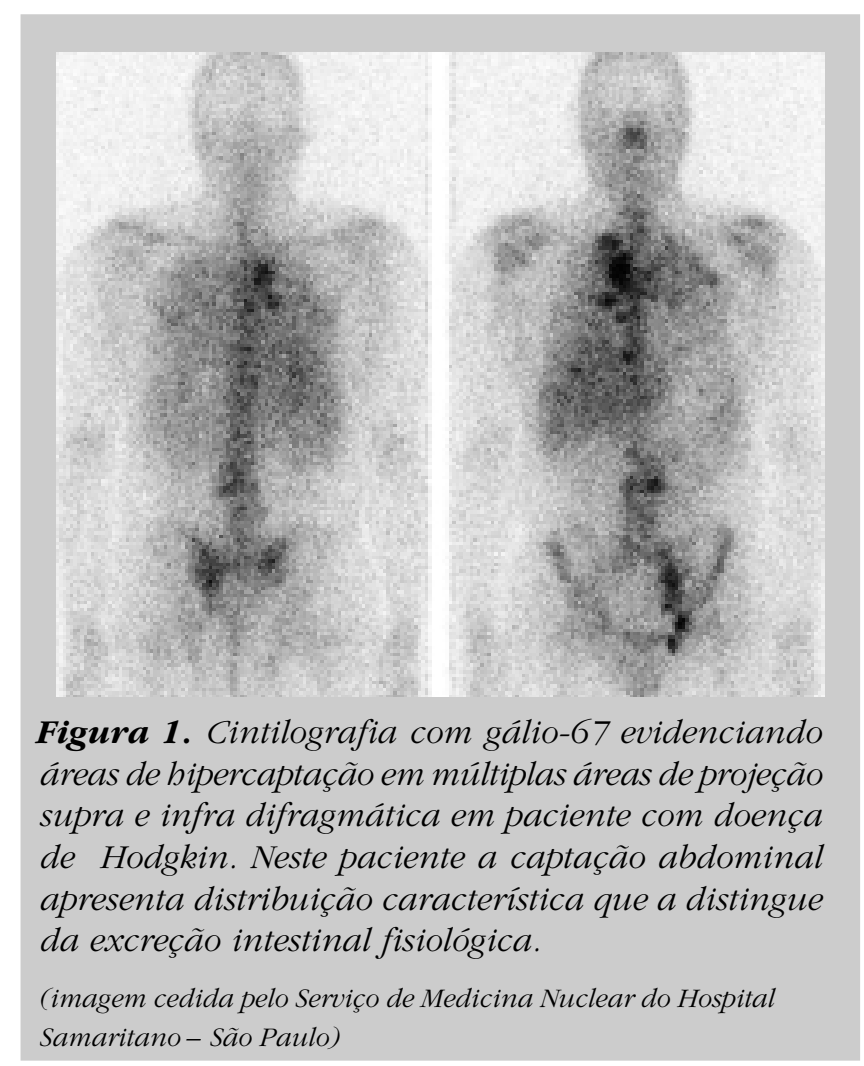

uso de altas doses de gálio-67. Alguns autores relatam bons resultados mesmo na avaliação de LNH de baixo grau, com sensibilidade de $79 \%$ nos estudos iniciais e de 89\% na detecção de recorrência em 57 pacientes (20), sensibilidade e especificidade de $89 \%$ e $92 \%$ em 34 pacientes (21).

\section{Acometimento extranodal}

Osso: A cintilografia óssea e com gálio-67 tem acurácia similar na detecção do linfoma ósseo (22). Porém, além de permitir a avaliação extra-óssea, estudos comparativos mostram que o Gálio-67 permite melhor acompanhamento da resposta terapêutica $(23,24)$. O padrão observado na cintilografia óssea é variável, incluindo lesões focais ou difusas com margens mal delimitadas, lesões fotopênicas e a acentuação da captação após tratamento (25). No linfoma ósseo primário o padrão observado na cintilografia óssea de 22 pacientes foi de hipercaptação com área central hipocaptante, enquanto que o gálio revelou maior captação na porção central da lesão (26).

A cintilografia pode ser associada a RM, permitindo um rastreamento de corpo inteiro para posterior confirmação diagnóstica de áreas suspeitas. Ao estudar 21 pacientes com acometimento ósseo, Stroszczynski relata que o Gálio-67 e a RM detectaram corretamente os 7 casos com linfoma ósseo primário, porém a sensibilidade do gálio-67 foi de apenas 62\% nos casos com envolvimento secundário. No acompanhamento por 3 a 5 meses após quimioterapia de 13 pacientes o gálio- 67 foi mais específico que a RM para doença em atividade, porém com menor sensibilidade (RM: sens. 90\% esp. 80\%, Ga: sens. 70\% esp. 93\%). O autor não relata se os casos de gálio-67 falso negativos no seguimento correspondiam aos pacientes com estudo basal também negativo (27).

Trato gastrointestinal: A atividade normal observada no fígado e baço, bem como a eliminação fisiológica do gálio-67 dificulta a análise abdominal, particularmente nos cólons. A captação fisiológica de gálio-67 pelo estômago é observada em cerca de 10\% dos pacientes e reduz a especificidade do método para detecção de linfoma gástrico (28). 
Sistema nervoso central: $O$ linfoma primário nesta região é pouco freqüente, com maior incidência em pacientes imunodeprimidos. A biópsia estereotáxica pode ser necessária para confirmação, porém métodos de imagem muitas vezes permitem um diagnóstico não invasivo. A medicina nuclear é utilizada no diagnóstico diferencial entre linfoma e toxoplasmose, havendo captação de gálio-67 e tálio-201 pelo primeiro. Dá-se preferência ao estudo tomográfico (SPECT) com tálio-201, porque este é avidamente concentrado pelos linfomas e apresenta menor captação em processos inflamatórios que o gálio-67. A sensibilidade descrita é próxima a 100\% com especificidade de 90 a 100\% (29, 30, 31).

\section{Seguimento e prognóstico}

O seguimento de pacientes é atualmente a principal indicação da cintilografia com gálio67. Mais de 50\% dos pacientes com linfoma mostram massa residual após tratamento, situação particularmente freqüente no acometimento mediastinal pela DH. A recorrência é observada em porcentagem significativamente menor (inferior a 20\%) (32, 33). As modalidades anatômicas (TC e RM) são capazes de mostrar a massa e sua morfologia, porém, pode ser difícil diferenciar entre tumor residual com células viáveis e áreas de fibrose/ necrose tumoral.

O gálio-67 não acumula em áreas de fibrose ou necrose, demonstrando-se em modelo animal que a sua captação é proporcional ao número de células viáveis após RT e QT (34). Por ser captado apenas pelas células viáveis, a cintilografia com gálio-67 é mais específica na diferenciação entre alterações seqüelares e doença residual (11). Porém, para que se possa correlacionar de forma segura a captação de gálio-67 com doença em atividade, é importante conhecer a avidez do tumor pelo radioisótopo antes do tratamento, evitando resultados falso negativos em tumores com baixa afinidade e que permanecem sem captação independente da resposta à quimioterapia (4).

Diversos autores relatam o valor da cintilografia com gálio-67 na avaliação de resposta terapêutica de linfomas. Anderson descreve uma acurácia de 96\% na definição ou exclusão de doença torácica ativa pelo gálio-67, com sensibilidade de 92\% em 72 pacientes (10). Weiner observou excelente correlação entre a cintilografia com gálio-67 negativa e a biópsia de massa residual no mediastino, mostrando fibrose/necrose, em 42 crianças tratadas, com maior dificuldade para avaliar lesões abdominais (35). De forma similar, Kostakoglu avaliou 30 pacientes com DH e massa residual submetida a biópsia, encontrando sensibilidade e especificidade do gálio-67 superiores às da TC na detecção de recorrência (gálio-67: sens 96\%, esp. 80\%, TC: sens 68\%, esp. 60\%) (36). A variação das dimensões da massa mostraram-se inferiores à cintilografia com gálio-67 para afastar doença em atividade após tratamento em 25 pacientes com DH e LNH, com especificidade do gálio-67 de 95\%, comparada a 57\% da TC e $55 \%$ do RX (14).

Além dos dados histopatológicos, os resultados da cintilografia com gálio-67 também foram correlacionados em diversos trabalhos com a evolução dos pacientes e recorrência em prazos determinados. Zinzani refere especificidade de $97 \%(30 / 31)$ e sensibilidade de 100\% (2/2) na predição de recorrência em 1 ano, Devizzi relata especificidade de 98\% em 47 pacientes $(37,38)$. A recorrência em 42 pacientes tratados e acompanhados em média por 2,5 anos foi muito menor em pacientes com gálio-67 negativo (5/ 22 em pacientes com massa residual e 1/11 em pacientes sem massa) do que nos pacientes com gálio-67 positivo (8/9) (18).

Front demonstrou em 43 pacientes com DH e 56 com LNH que, além do alto valor preditivo negativo, o SPECT com gálio-67 tem bom valor prognóstico. $O$ estudo negativo indica maior intervalo livre de doença $(\mathrm{p}<0,05$ para $\mathrm{DH}$ e $<$ 0,0001 para LNH), não havendo informação prognóstica significativa na TC (39). Ao analisar 139 pacientes com LNH de grandes células pós tratamento, o gálio-67 teve 90\% de sensibilidade na predição de recorrência em 36 pacientes (40).

Estudos comparativos mostram resultados semelhantes da RM e gálio-67 na avaliação de massas residuais $(37,38)$. Apesar da RM mostrar resultados comparáveis ao gálio-67, a sua normalização ocorre mais tardiamente, cerca de 6 meses após a resolução da captação de gálio- 
67 (41), sendo descrito que o padrão dos tumores ativos e tecido necrótico podem ser semelhantes na fase pós terapêutica precoce (42). A RM é indicada particularmente se o estudo com gálio-67 pré-tratamento for negativo.

Os resultados obtidos pela cintilografia com gálio-67 para seguimento de linfoma não são, no entanto, homogêneos. Bogart relata baixo valor preditivo negativo do gálio-67 no acompanhamento, visto que 10 de 29 pacientes com DH e cintilografia pósquimioterapia negativa apresentaram recorrência (43). Em 34 pacientes com DH ou LNH grau alto/intermediário a RM alterada apresentou especificidade de 90\% para recorrência, porém pelos critérios utilizados a sensibilidade foi de apenas $45 \%$ para controle e prognóstico. Nos mesmos pacientes o SPECT com gálio-67 apresentou apenas 33\% de sensibilidade, porém não foram realizados estudos pré tratamento que comprovassem a avidez do tumor (44).

O valor da cintilografia ao término do tratamento é bem aceito pela maioria dos autores. Mais recentemente tem sido proposto que a variação de captação de gálio-67 na vigência da quimioterapia tenha também valor prognóstico e poderia justificar uma mudança precoce no esquema terapêutico. Kaplan constatou que apenas $24 \%$ dos pacientes com LNH de alto grau com captação persistente após 4 a 6 ciclos do tratamento tiveram remissão completa, comparado a 55\% dos que não tinham captação. Houve também diferença na sobrevida dos pacientes (óbito de 59\% x 25\%) (45). Estudos precoces com gálio-67 foram empregados para predizer resposta a quimioterapia em pacientes com LNH de alto grau após o primeiro ciclo $(n=51)$ e entre o 3 e 4 ciclos $(n=97)$. Falha no tratamento foi observada em $71 \%$ dos pacientes com estudo positivo e 19\% dos pacientes com estudos negativos após o primeiro ciclo, 74\% dos pacientes com gálio positivo e $37 \%$ dos pacientes com gálio negativo após 3 a 4 ciclos. Como resultado de análise multifatorial, os dois tempos foram considerados preditores independentes de sobrevida, podendo indicar a necessidade de substituição precoce do esquema terapêutico (46).

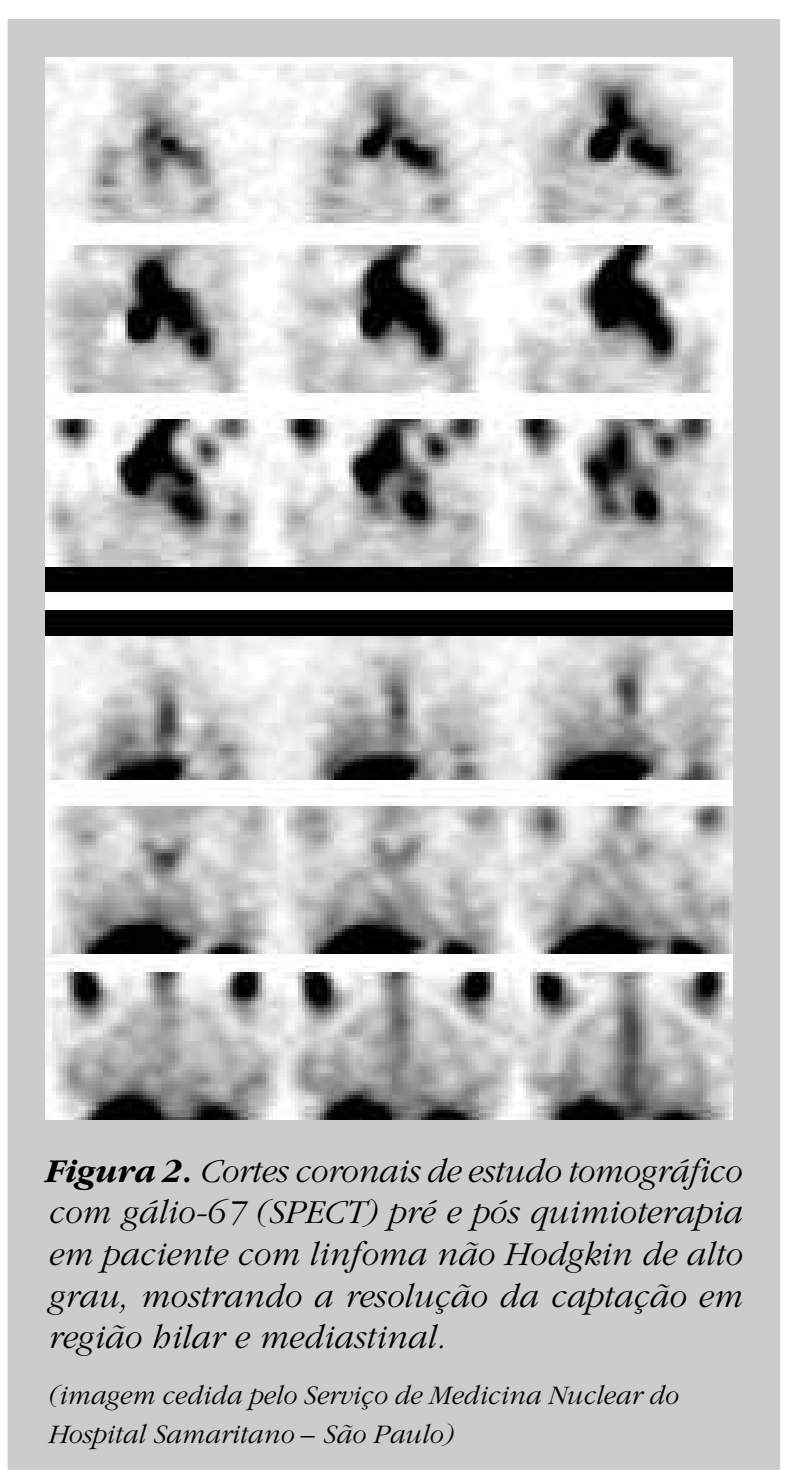

\section{Estudos com Fluordeoxiglicose ( ${ }^{18}$ F-FDG)}

\section{Aspectos gerais}

Os estudos com ${ }^{18} \mathrm{~F}$-fluordeoxiglicose $\left({ }^{18} \mathrm{~F}\right.$ FDG), recentemente introduzidos em nosso meio, vêm apresentando grande destaque no estadiamento e seguimento de linfoma, com alta acurácia na detecção de acometimento linfonodal e extranodal. O método baseia-se na administração de um análogo da glicose, marcada com o flúor-18. O flúor-18 emite pósitrons, partículas que sofrem aniquilação e emitem radiação gama detectada por aparelhos dedicados (PET) ou câmaras de cintilação com sistemas de coincidência. As imagens 
tomográficas obtidas após a administração do radiofármaco refletem a taxa metabólica regional (a ${ }^{18}$ F-FDG é fosforilada mas não segue a via glicolítica, ficando aprisionada no meio intracelular). Alto metabolismo e conseqüente captação de ${ }^{18}$ F-FDG é observado em várias neoplasias e alguns processos inflamatórios.

\section{Avaliação inicial e estadiamento}

A alta taxa metabólica celular dos linfomas permite que a captação de ${ }^{18}$ F-FDG seja empregada para o estadiamento, com sensibilidade próxima ou superior a da TC (47). Alguns estudos sugerem que a sensibilidade seja maior em pacientes com LNH de alto grau $(33,48)$, porém o método também é aplicado com sucesso no estadiamento de DH e LNH de baixo grau (49, 50). O PET com FDG mostrou sensibilidade próxima a TC e especificidade significativamente maior na detecção de linfonodos que a TC em 50 pacientes (DH: sens 86\% esp. 96\%, LNH: sens 89\% esp. 100\%) (50). Na detecção de acometimento hilar e mediastinal com PET-FDG, Bangerter relata sensibilidade de $96 \%$ e especificidade de $94 \%$ em 89 pacientes (51).

A detecção de maior número de linfonodos que a TC pode implicar em mudança no estádio, sendo relatado por Moog o supra-estadiamento em 8\% de 60 pacientes (49). O estudo de corpo inteiro com PET-FDG foi também comparado ao conjunto dos métodos de estadiamento convencional (TC, US, RM, cintilografia óssea, biópsia de medula, biópsia hepática, laparotomia) em 44 pacientes com suspeita de DH. O PETFDG foi positivo em $86 \%$ e modificou o estádio em 14\% dos pacientes (aumentou em 5 e reduziu em 1 caso) (52). O maior número de linfonodos detectado ao PET que a TC não acarreta obrigatoriamente uma mudança de estádio ou conduta (53), porém em estudo recente o estadiamento e a conduta foram modificados em 50\% dos pacientes (32\% com aumento e 18\% com redução do estádio) (54). Ressalta-se que, além da alta sensibilidade na detecção de linfonodos, O PET tem alta acurácia na avaliação de acometimento extranodal e no seguimento pós tratamento.

A sensibilidade do PET com FDG parece ser superior a do gálio-67. Estudo comparativo mostra maior sensibilidade da PET-FDG que o SPECT com gálio-67 em 47 pacientes com LNH e 15 pacientes com $\mathrm{DH}$, em todos os tipos histológicos da doença (detecção de lesões: 100\% x 69\%, detecção de pacientes: 100\% x 81\%) (55). Outro estudo com 46 pacientes (36 LNH e 10 DH) mostrou sensibilidade na detecção de lesões de $96 \%$ para o PET-FDG e $82 \%$ para o gálio-67, com sensibilidade para pacientes de, respectivamente, $100 \%$ e $89 \%$ (56). No estudo de Wirth os resultados de PET-FDG foram mais próximos aos do gálio-67, com sensibilidade de $89 \%$ para o PET-FDG e $81 \%$ para o gálio-67, com 14\% dos 93 pacientes supra-estadiados por cada um dos métodos (57).

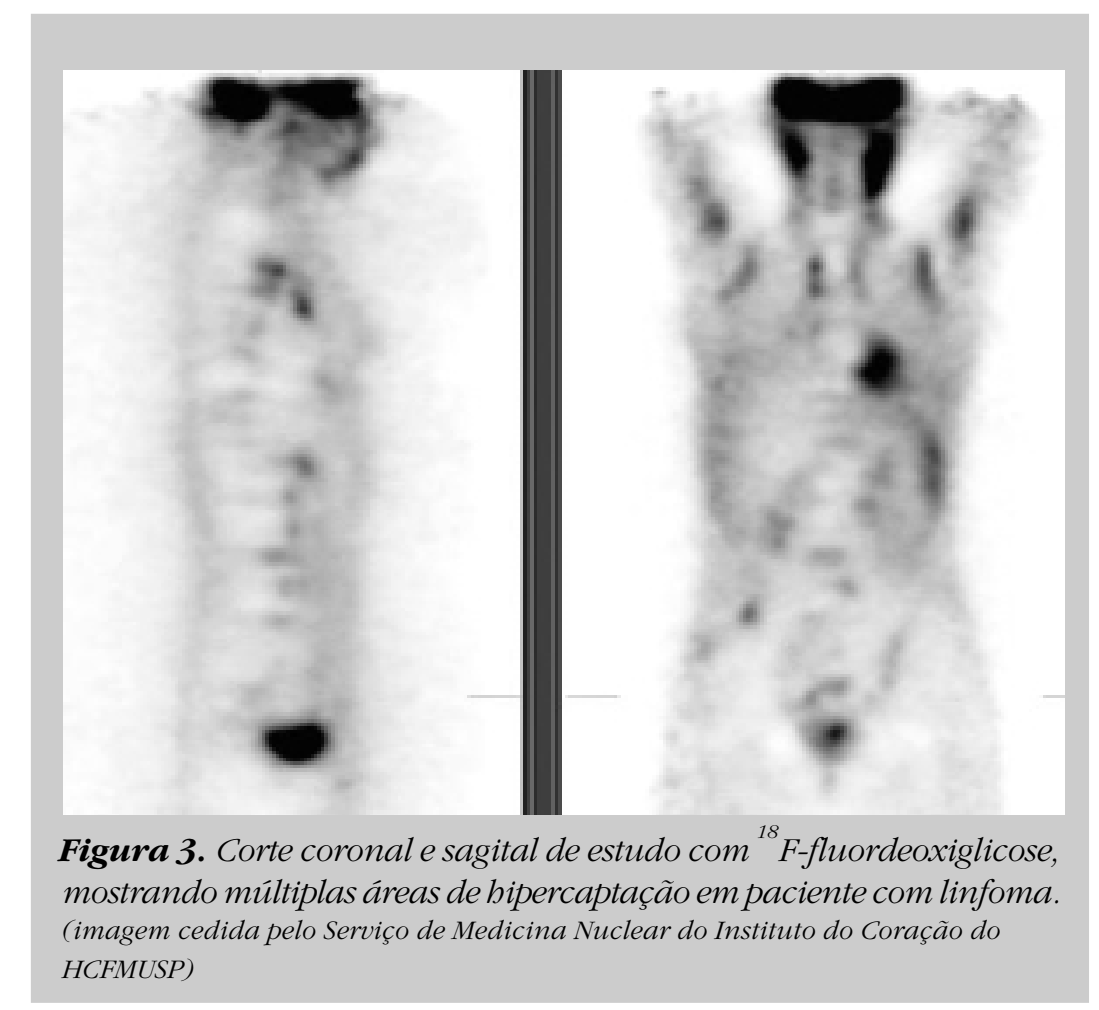


Acometimento extranodal - Moog relata excelentes resultados do PET com FDG na avaliação inicial de doença extranodal em 81 pacientes ( $\mathrm{LNH}=43, \mathrm{DH}=38)$, determinando-se mudança do estádio em 13 pacientes. No total, 42 lesões extranodais foram identificadas tanto pela TC como pelo PET-FDG, 7 detectadas apenas pela TC ( 5 falso positivas, 1 infiltração renal, 1 sem seguimento) e 24 lesões só vistas pelo PET-FDG ( 1 falso positivo, 14 lesões confirmadas, 9 sem seguimento). Das 14 lesões confirmadas 9 eram medulares, 3 em baço e 2 em outras localizações (58).

Jerusalem, estudando 60 pacientes (23 LNH alto grau, $21 \mathrm{LNH}$ baixo grau, $16 \mathrm{DH})$, mostra também o maior impacto do PET-FDG no estadiamento extra-nodal. Apesar da detecção de maior número de linfonodos que a TC, o PET mudou o estádio em apenas 2 pacientes, sem determinar mudanças no tratamento. A concordância do PET e TC para lesões hepáticas e esplênicas foi boa, porém sem confirmação histológica nos casos discordantes, havendo maior sensibilidade do PET na detecção de infiltração da medula óssea (53).

Na detecção de acometimento ósseo o PETFDG parece ser superior a cintilografia óssea, com maior número de lesões detectadas e maior especificidade. Dois de 56 pacientes tiveram lesões detectadas pelos dois métodos, 5 apenas com PET ( 3 confirmadas, 2 sem seguimento) e 5 apenas com MDP (3 não confirmadas, $2 \mathrm{sem}$ seguimento) (59).

A captação difusa e discreta de ${ }^{18}$ F-FDG na medula óssea deve ser interpretada com cautela, podendo ser causada por ativação medular (mais freqüente no primeiro mês após quimioterapia ou uso de fatores de crescimento). Sugere-se a manutenção da biópsia de medula como principal método diagnóstico, com a RM indicada para casos específicos. A literatura mostra resultados concordantes do PET-FDG com a biópsia de medula óssea em 78\% de 50 pacientes (38 DH e $12 \mathrm{LNH}$ ), sendo 13 positivos e 26 negativos. Em 4 pacientes suspeitou-se de biópsia falsa negativa, por ter sido realizada distante do local com captação de PET, em outros 4 pacientes o PET foi considerado falso positivo. Dentre 3 casos com PET falso negativo,
2 também não apresentavam captação nos linfonodos acometidos (60). O estudo de Jerusalem também mostra biópsia negativa em 4 de 18 casos com PET-FDG positivo, ressaltando que a biópsia não foi efetuada no sítio suspeito (53).

Uma situação específica na qual relata-se baixa sensibilidade do PET-FDG é o linfoma de células B associado ao tecido linfóide de mucosas (MALT). Todos os estudos realizados em um total de 10 pacientes foram negativos (61).

\section{Seguimento e prognóstico}

Assim como o gálio-67, vários estudos abordaram o uso do PET-FDG na caracterização metabólica da massa residual pós tratamento, para diferenciar tumor residual de tecido fibroso/necrose. Os estudos mostram uma excelente sensibilidade para doença residual, com alguns casos falso positivos determinados principalmente por processo inflamatório.

No estudo de Dewit o PET com FDG apresentou sensibilidade de 100\% (8/8) e especificidade de $73 \%(17 / 22)$ para predição de recorrência após tratamento em pacientes com massa residual, com período médio de acompanhamento de 14 meses (62). A sensibilidade de 100\% (15/15) também é relatada por Cremerius, com especificidade de 92\% (11/ 12) no seu grupo de 27 pacientes (48). Bangerter, avaliando 36 pacientes com massa residual, observou sensibilidade de 71\% (5/7) e especificidade de 86\% (25/29) na predição de recorrência durante 25 meses (63) e Mikhaell, estudando 32 pacientes, obteve sensibilidade de $80 \%$ (8/10) e especificidade de $95 \%(21 / 22)$ com 38 meses de seguimento (64). Outro estudo recente com PET-FDG mostrou sensibilidade de 100\% (26/26) e especificidade de 80\% (56/69) para a detecção de doença residual ou recorrência em pacientes submetidos a quimioterapia e monitorados durante pelo menos 1 ano (65).

O estudo de Jerusalem resultou em uma sensibilidade e especificidade de, respectivamente, 43\% (6/14) e 100\% (40/40) na predição de recorrência em 54 pacientes, 24 deles com massa residual. É interessante observar que, das 5 recorrências observadas nos 
casos com PET negativo e TC alterada, 4 ocorreram fora da massa. Outras 3 recorrências com PET negativo foram observadas em pacientes sem massa na TC. Além da recorrência, os autores mostram a variação de sobrevida de 1 ano em função da captação de ${ }^{18}$ F-FDG. Os pacientes com PET negativo apresentavam maior chance de se encontrarem livres de doença em 1 ano do que os pacientes com estudo alterado (86 x 0\%) e maior sobrevida (92 x 50\%), tendo este dado maior valor que a presença de massa residual na TC (livres de doença $88 \times 62 \%$, sobrevida $95 \times 77 \%$ ) (66).

Além de fatores relacionados ao próprio linfoma e tratamento realizado, o tipo de equipamento empregado pode interferir no resultado. Empregando um equipamento de PET de baixa resolução, Maisey refere uma sensibilidade de apenas $50 \%$ e especificidade de 69\%, comparados a RM com valores de, respectivamente, $45 \%$ e $74 \%$ (33)

As causas de estudos falso positivo incluem pneumonite actínica (48), pneumonia e trauma (62), sendo importante correlacionar a captação de ${ }^{18}$ F-FDG com a localização da massa na TC ou RM $(66,67)$. Assim como os processos inflamatórios, a hiperplasia de timo, causa

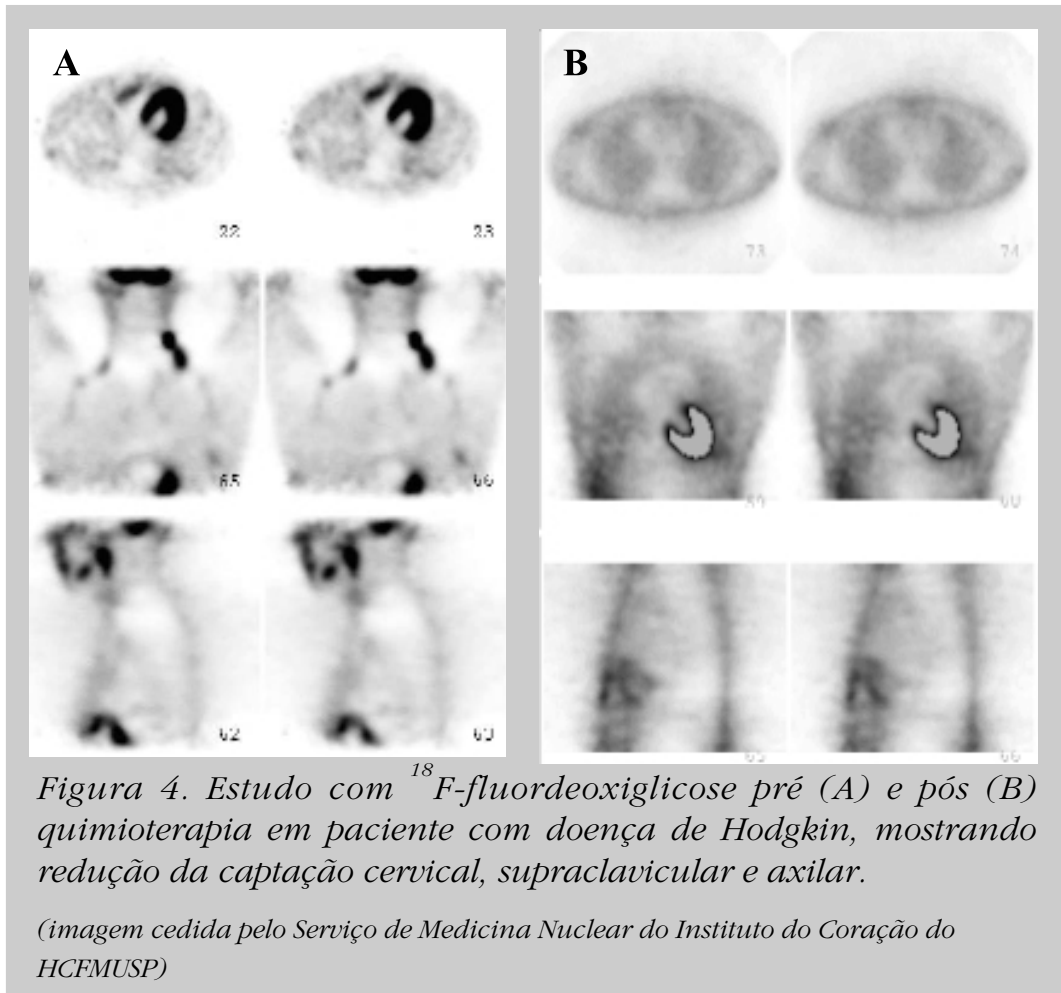

reconhecida de aumento da captação de gálio67 no acompanhamento pós tratamento de pacientes jovens, pode ocasionalmente levar a hipercaptação de ${ }^{18}$ F-FDG $(51,68)$.

\section{Estudos com outros radiofármacos / radioisótopos}

\section{Cintilografia com tálio-201}

O tálio-201 é um radioisótopo análogo ao potássio, captado através da bomba $\mathrm{Na} / \mathrm{K}$ ATPase. Apesar de ser mais empregado em estudos de perfusão miocárdica, o tálio-201 também tem sido aplicado em oncologia, pois a sua captação reflete a viabilidade e atividade metabólica celular (69).

Alguns autores relatam maior captação de tálio-201 do que de gálio-67 em LNH de baixo grau, ainda não estando claramente definido o papel deste isótopo neste subgrupo de pacientes. Waxman relata um aumento na detecção de lesões de 32\% com gálio-67 para 100\% com tálio-201 em 9 pacientes com LNH de baixo grau, não havendo diferença significativa na sensibilidade nos demais 27 pacientes com DH ou LNH de grau alto/ intermediário (19). A análise combinada das cintilografias com tálio-201 e com gálio-67 também resultou em maior sensibilidade no estudo de 33 pacientes, com maior impacto do tálio-201 nos pacientes com LNH de baixo grau ou intermediário (sensibilidade: gálio- $67=67 \%$, tálio-201=73\%, combinada=82\%) (70).

Descreve-se a redução mais rápida da captação de tálio-201 que a de gálio-67 no acompanhamento após tratamento de linfomas ósseos, sugerindo sua indicação no acompanhamento de resposta das lesões. Porém, para que se valorizem os dados obtidos no seguimento é importante que se demonstre a captação em estudo basal, da mesma forma que nos estudos com gálio-67 (71). 


\section{Cintilografia com Sestamibi-Tc99m}

O sestamibi marcado com tecnécio-99m é utilizado em estudos de perfusão miocárdica e, de forma análoga ao tálio-201, pode ser captado em linfomas e outros tumores. A captação se faz por difusão passiva, dependente do potencial elétrico através da membrana celular e mitocondrial.

Um ponto de interesse na investigação de tumores com sestamibi-Tc99m é que a sua eliminação celular se faz através da glicoproteína P (Pgp), mesmo sistema responsável pelo transporte para fora da célula de diversos quimioterápicos. É relatado que o grau de captação a velocidade de clareamento do sestamibi-Tc99m estão associados à resistência a multidrogas (67).

\section{Cintilografia com Octreotide}

O octreotide é um análogo da somatostatina, com maior meia-vida plasmática, marcado com indio-111 para realização da cintilografia. Após sua administração o radiofármaco se liga a receptores de somatostatina, encontrados em tumores neuroendócrinos, outros tumores malignos (incluindo linfomas) e ocasionalmente em infiltrados inflamatórios.

Apesar do interesse recente, o custo do procedimento é alto e a detecção de lesões infra-diafragmaticas é prejudicada pela captação fisiológica do octreotide em fígado, rins e baço. Nos casos de LNH o método provavelmente não tenha aplicação, sendo os melhores resultados obtidos na $\mathrm{DH}$ com lesões supra-diafragmáticas (sem grandes vantagens em relação ao gálio-67). A sensibilidade descrita varia de 29 a 37\% para LNH e até $70 \%$ para $\mathrm{DH}(72,73)$. Mesmo utilizando SPECT, apenas $48 \%$ das lesões foram detectadas em 10 pacientes (60\% acima e 30\% infra-diafragmáticas) (74). Estudo de 90 pacientes com LNH mostra sensibilidade de $66 \%$, sendo $72 \%$ para lesões supradiafragmáticas e $52 \%$ para infra-diafragmáticas (75). Melhores resultados são descritos pelo mesmo autor em 56 pacientes com DH, com positividade de $98 \%$, detecção de novas lesões em 36\%, mudança de estádio em 21\% e de conduta em 13\% dos pacientes (76).

\section{Cintilografia de medula óssea}

Os principais radiofármacos utilizados para a caracterização funcional da medula são os colóides marcados com tecnécio-99m (fagocitados por macrófagos) e os anticorpos monoclonais anti-granulócitos marcados (que se ligam à antígenos da membrana celular). Um achado freqüente nos pacientes com linfoma é a expansão medular periférica, que ocorre em 33 a 81\% dos casos (77). O método pode ser indicado para avaliar a substituição focal da medula (podendo inclusive orientar sítios de biópsia), sendo mais difícil a identificação de acometimento medular difuso $(78,79)$.

A ressonância magnética mantém-se como o método de imagem com maior acurácia na detecção de envolvimento medular, sendo proposta a cintilografia de medula como uma alternativa para rastreamento de corpo inteiro. A comparação entre RM e cintilografia de medula óssea com anticorpos anti-granulócitos marcados com tecnécio-99m mostra concordância em 28/32 pacientes (2 sem seguimento, 1 falso positivo na RM, 1 falso negativo na cintilografia) (78). Houve concordância entre a cintilografia com anticorpos anti-granulócitos-Tc99m e a biópsia em 69/103 pacientes com DH e LNH, com menor sensibilidade para LNH de baixo grau, particularmente quando o envolvimento medular era difuso (DH: sens. 100\% esp. 84\%, LNH baixo grau: sens. 60\%, alto grau sens. 93\% esp. 84\%). Apesar dos 5 casos falso negativos e 15 casos falso positivos, aproximadamente metade das lesões detectadas apenas pela cintilografia foi comprovada na evolução (80). Outro estudo, empregando colóides marcados com tecnécio-99m, mostra menor sensibilidade da cintilografia (DH: 50\%, LNH alto grau: 75\%, LNH baixo grau: 30\%) (81).

\section{Cintilografia com anticorpos monoclonais marcados}

Ao lado dos anticorpos antigranulócitos, previamente citados para o estudo de medula óssea, diversos anticorpos monoclonais dirigidos contra antígenos de expressão linfocitária (CD20, CD21, CD22, CD37) foram desenvolvidos. Os anticorpos monoclonais podem ser marcados com emissores de radiação beta, como o iodo- 
131 e ítrio-90, isótopos altamente ionizantes empregados com finalidade terapêutica. Os resultados, apesar da casuística ainda pequena, são promissores, com resposta em até 50-80\% dos pacientes refratários a outras modalidades de tratamento (82).

O anticorpo monoclonal anti-CD20 (rituximab) é uma opção recentemente aprovada pelo FDA americano no tratamento para pacientes com linfoma de células B refratário ou recorrente. Empregando o anticorpo não radioativo ocorre remissão em 60\% dos casos de recorrência de linfoma folicular (5 a 10\% remissão completa). Ao ser marcado com iodo-131 e ítrio90 a remissão alcança 75 a 80\% (35 a 40\% remissão total) ou valores de 85 a 90\% (75 a 80\% de remissão completa) quando altas doses são associadas ao transplante de medula óssea (83). Em estudos de fase I-II com anticorpo anti-CD20 marcado com itrío-90 a resposta foi de 64 a 67\% em pacientes com LNH de baixo grau ou intermediário (84).

\section{Contribution of nuclear medicine in lymphomas approach}

Marcelo T. Sapienza, Marília M. S. Marone, Carlos S. Chiattone

\section{Abstract}

Nuclear medicine procedures allow functional characterization of organs and tissues, that can be compared to the anatomical evaluation provided by computed tomography, magnetic resonance imaging and ultrasonography. Functional information are usefull not only in diagnosis and staging, but mainly in the followup and evaluation of the therapeutic response of lymphoma patients. Persistent or residual mediastinal mass and and infiltration of small nodes are some examples of situations in which purely anatomical evaluation is insufficient. The main methods in use are scintigraphy with galium-67, a transferrin avid tracer, and PET with fluoro-desoxyglucose, indicated for the metabolic evaluation of tissues. Other tracers are also described, as thallium-201, 99mTcsestamibi, and 111In-octreotide. Mechanisms of uptake, indications, limitations and results of the literature are and discussed in this review. Rev.bras.hematol.hemoter., 2001, 23 (2): 79-92

Key words: Lymphoma, Scintigraphy, Gallium, PET

\section{Referências Bibliográficas}

1. Edwards CL, Hayes RL. Tumor scanning with Ga-67 citrate. J Nucl Med. 1969; 10: 103-5.

2. Larson SM, Greenbaum Z, Rasey JS. The role of transferrins in gallium uptake. Int J Nucl Med Biol. 1981; 8: 257-66.

3. Nejmeddine F, Raphael M, Martin A, LeRoux G, Moretti JL, Caillat-Vigneron N. ${ }^{67} G a$ scintigraphy in B-cell non-Hodgkin's lymphoma: Correlation of 67-Ga uptake with bistology and transferrin receptor expression.

J Nucl Med 1999; 40: 40-5.

4. Van Amsterdam JAG, Kluin-Nelemans JC, VanEck-Smit BLF, Pauwels EKJ. Role of 67Ga scintigraphy in localization of lymphoma. Ann Hematol 1996; 72: 202-7.

5. Hattner RS, White DL. Gallium-67/stable gadolinium anatagonism: MRI contrast agent markedly alters the normal biodistribution of gallium-67. J Nucl Med 1990; 31:1844-6.

6. Even Sapir E, Bar Shalom R, Israel O. Singlephoton emission computed tomography quantitation of gallium citrate uptake for the differentiation of lymphoma from benign bilar uptake. J Clin Oncol 1995; 13:942-6.

7. Drossman SR, Schiff RG, Kronfeld GD, McNamara J, Leonidas JC. Lymphoma of the mediastinum and neck: evaluation with $\mathrm{Ga}$ 67 imaging and CT correlation. Radiology 1990; 174: 171-5.

8. Luker GD, Siegel MJ. Mediastinal Hodgkin disease in children: response to therapy. Radiology 1993; 189: 737-40.

9. Peylan-Ramu N, Haddy TB, Jones E, Horvath K, Adde MA, Magrath IT. High frequency of benign mediatinal uptake of gallium-67 after completion of chemotherapy in children with bigh grade non-Hodgkin's lymphoma. J Clin Oncol 1989; 7: 1800-6.

10. Anderson KC, Leonard RC, Canellos GP, Skarin AT, Kaplan WD. High dose gallium imaging in lymphoma. Am J Med 1983; 75: 327-31.

11. Front D, Bar-Shalom $\mathrm{R}$, Israel O. The continuing clinical role of gallium 67 scintigraphy in the age of receptor imaging. Sem Nucl Med 1997; 27: 68-74.

12. Kaplan WD. Residual mass and negative 
gallium scintigraphy in treated lymphoma: when is gallium really negative (ed.). $\mathbf{J}$ Nucl Med 1990; 31: 369-71.

13. Van der Wall H, McLaughlin AF, Southee AE. Gallium scintigraphy in tumor diagnosis and management. in: Murray IPC, Ell PJ, eds. Nuclear Medicine in Clinical Diagnosis and Treatment (2ed) Edinburgh: Churchill Livingstone; 1998 p 813-29.

14. Israel O, Front D, Lam M, Ben-Haim S, Kleinhaus U, BenSachar M, Robinson E, Kolodny GM. Gallium-67 imaging in monitoring lymphoma response to treatment.

Cancer 1988; 61: 2439-43.

15. Bekerman C, Hoffer PB, Bitran JD. The role of gallium-67 in the clinical evaluation of cancer. Sem Nucl Med 1985; 15: 72-103.

16. Tumeh SS, Rosenthal DS, Kaplan WD, English RJ, Holman BL. Lymphoma: evaluation with Ga-67 SPECT. Radiology 1987; 164:111-4.

17. Hagemeister F, Fesum S, Larriki L, Hayme E. Role of gallium scan in Hodgkin's disease. Cancer 1990; 65:1090-6.

18. Delcambre C, Reman O, Henry Amar M, Peny AM, Macro M, Cheze S, Génot JY, Tanguy A, Switsers O, Van HL, Couëtte JE, Leporrier M, Bardet S. Clinical relevance of gallium-67 scintigraphy in lymphoma before and after therapy. Eur J Nucl Med 2000; 27:2176-84.

19. Waxman AD, Eller D, Ashook G, Ramanna L, Brachman M, Heifetz L, McAndrews P, Bierman H, Taub R, Avedo M, Wall F. Comparison of Ga-67 and thallium-201 scintigraphy in peripheral and intrathoracic lymphoma. J Nucl Med 1996; 37:46-50.

20. Ben Haim S, Bar Shalom R, Israel O. Utility of gallium-67scintigraphy in low-grade nonHodgkin's lymphoma. J Clin Oncol 1996; 14: 1936-42.

21. Sandrock D, Lastoria S, Magrath IT, Neumann $\mathrm{RD}$. The role of gallium-67tumour scintigraphy in patients with small, non-cleaved cell lymphoma. Eur J Nucl Med 1993; 20:119-22.

22. Mouratidis B, Gilday DL, Ash JM. Comparison of bone and Ga67 scintigraphy in the initial diagnosis of bone involvement in children with malignant lymphomas. Nucl Med Commun. 1994 ; 15: 144-7.
23. Moon TY, Kim EE, Kim YC, Chung JK, Kim BS, Lee SH, Kim KI, Podoloff DA. Comparison of nuclear bone and gallium scans in the therapeutic evaluation of bone lymphoma. Clin Nucl Med 1995; 20:721-4.

24. Bar-Shalom R, Israel O, Epelbaum R, Haim $\mathrm{N}$, Ben-Arush M, Ben-Sachar M, Leviov M, Gaitini D, Ben-Haim S, Even Sapir E. Gallium67 scintigraphy in lymphoma with bone involvement. J Nucl Med 1995; 36: 446-50.

25. Van Nostrand DV, Madewell JE, McNiesh LM, Kyle RW, Sweet D. Radionuclide bone scanning in giant cell tumor. J Nucl Med 1986; 27: 329-38.

26. Leeson MC, Makely JT, Carter JR, Krupco T. The use of radioisotope scans in the evaluation of primary lymphoma of bone. Orthop Rev 1989; 18:410-6.

27. Stroszczynski C, Oellinger J, Hosten N, Spahn G, Amthauer H, Ricke J, Hoffman KT, Eichstaedt H, Ludwig WD, Felix R. Staging of malignant lymphoma of the bone: comparison of ${ }^{67}$ a scintigraphy and MRI. $\mathbf{J}$ Nucl Med 1999; 40: 387-93.

28. MacMahon H, Vyborny C, Sephardari S, Kirchner P, Ryan J. Gallium accumulation in the stomach. A frequent incidental finding. Clin Nucl Med 1985; 10: 719-23.

29. Lorbeyboym M, Estok L, Machac J, Germano I, Sacher M, Feldman R, Wallach F, Dorfman D. Rapid differential diagnosis of cerebral toxoplasmosis and primary central nervous system lymphoma by thallium SPECT.J Nucl Med 1996; 37: 1150-4.

30. Ruiz A, Ganz WI, Post JD, Camp A, Landy $\mathrm{H}$, Mallin W, Sfakianakis GN. Use of thallium201 brains SPECT to differentiate cerebral lymphoma from toxoplasma encephalitis in AIDS patients AJNR 1994; 15: 1885-94.

31. Lee VW, Antonacci V, Tilak S, Fuller JD, Cooley TP. Intracranial mass lesions: sequential thallium and gallium scintigraphy in patients with AIDS. Radiology 1999; 211: 507-12.

32. Canellos GP. Residual mass in lymphoma may not be residual disease (editorial). $\mathbf{J}$ Clin Oncol 1988; 6:931.

33. Maisey NR, Hill ME, Webb A, Cunningham D, Flux GD, Padhani A, Ott RJ, Norman A, 
Bishop L. Are 18-fluorodeoxyglucose postitron emission tomography and resonance imaging useful in the prediction of relapse in lymphoma residual masses? Eur J Cancer 2000; 36: 200-6.

34. Iosilevsky G, Front D, Bettan L, Hardoff R, Ben-Arieh Y. Uptake of gallium67-citrate and [2-3H]deoxyglucose in the tumor model, followin chemotherapy and radiotherapy. $\mathbf{J}$ Nucl Med 1985; 26: 278-282.

35. Weiner M, Leventhal B, Cantor A, Wimmer R, Bery DL, Cook B, Ducos R. Gallium-67 scans as an adjunct to computed tomography scans for the assessment of a residual mediastinal mass in pediatric patients with Hodgkin's disease. A pediatric oncology group study. Cancer 1991; 68: 2478-80.

36. Kostakoglu L, Yeh SD, Portloch C, Heelan R, Yao TJ, Niedzwiecki D, Larson SM. Validation of gallium-67-citrate single photon emission computed tomography in biopsy-comfirmed residual Hodgkin's disease in the mediastinum. J Nucl Med 1992; 33:345-50.

37. Zinzani PL, Zompatori M, Bendani M, Battista G, Fanti S, Barbieri E, Gherlinzoni F, Rimondi MR, Frezza G, Pisi P, Merla E, Gozzetti A, Canini R, Monetti N, Babini L, Tura S. Monitoring bulky mediastinal disease with gallium-67, CT scan and magnetic resonance imaging in Hodgkin's disease and high grade non-Hodgkin's lymphoma. Leuk Lymphoma 1996; 22: 131-5.

38. Devizzi L, Maffioli L, Bonfante V, Viviani S, Balzarini L, Gasparini M, Valagussa P, Bombardieri E, Santoro A, Bonadonna G. Comparison of gallium scan, computed tomography, and magnetic resonance in patients with mediastinal Hodgkin's disease.

Ann Oncol 1997; 8:53-6.

39. Front D, Ben-Haim S, Israel O, Epelbaum R, Haim N, Evensapir E, Kolodny GM, Robinson E. Lymphoma: predictive value of Ga-67 scintigraphy after treatment. Radiology 1992; 182: 359-63.

40. Weeks JC, Yeap BY, Canellos GP, Shipp MA. Value offollow-up procedures in patients with large-cell lymphoma who achieve a complete remission. J Clin Oncol 1991; 9: 1196-203.

41. Abrahamsen AF, Lien HH, Aas M, Wideren
M, Hager B, Kvaloy S, Elgjo R, Nome O. Magnetic resonance imaging and 67 gallium scan in mediastinal malignant lymphoma: a prospective pilot study. Ann Oncol 1994; 5: 433-6.

42. Rahmouni A, Tempany C, Jones R, Mann R, Yang A, Zerhouni E. Lymphoma: monitoring tumor size and signal intensity with MR imaging. Radiology 1993; 188: 445-51.

43. Bogart JA, Chung CT, Mariados NF, Vermont AI, Lemke SM, Grethlein S, Graziano SL. The value of gallium imaging after therapy for Hodgkin's disease. Cancer 1998; 82: 754-9.

44. Hill M, Cunningham D, MAcVicar D, Roldan A, Husband J, McCready R, Mansi J, Milan $\mathrm{S}$, Hickish T. Role of magnetic resonance imaging in predicting relapse in residual masses after treatment of lymphoma. J Clin Oncol 1993; 11: 2273-8.

45. Kaplan WD, Jochelson MS, Herman TS, Nadler LM, Stomper PC, Takvorian T, Andersen JW, Canellos GP. Gallium-67 imaging: a predictor of residual tumor viability and clinical outcome in patients with diffuse large-cell lymphoma. J Clin Oncol 1990; 8: 1966-70.

46. Front D, Bar-Shalom R, Mor M, Haim N, Epelbaum R, Frenkel A, Gaitini D, Kolodny GM, Israel O. Aggressive Non-Hodgkin lymphoma: Early prediction of outcome with 67-Ga scintigraphy. Radiology 2000; 214: 253-7.

47. Hoh CK, Glaspy J, Rosen P, Dahlbom M, Lee SJ, Kunkel L, Hawkin RA, Maddahi J, Phelps ME. Whole-body FDG-PET imaging for staging of Hodgkin's disease and lymphoma. J Nucl Med 1997; 38: 343-8.

48. Cremerius U, Fabry U, Neuerburg J, Zimny M, Osieka R, Buell U. Positron emission tomography with 18F-FDG to detect residual disease after therapy for malignant lymphoma. Nucl Med Commun 1998; 19: 1055-63.

49. Moog F, Bangerter M, Diedrichs CG, Guhlmann A, Kotzerke J, Merkle E, Kolokythas O, Herrmann F, Reske SN. Lymphoma: role of whole-body 2-deoxy-2(F18) fluoro-d-glucose (FDG) PET in nodal staging. Radiology 1997; 302: 795-800. 
50. Stumpe KDM, Urbinelli M, Steinert HC, Glanzmann CH, Buck A, von Schulthess GK. Whole-body positron emission tompography using fluorodeoxyglucose for staging of lymphoma: effectiveness and comparison with computed tomography. Eur J Nucl Med 1998; 25: 721-8.

51. Bangerter M, Kotzerke J, Griesshammer M, Elsner K, Reske SN, Bergmann L. Positron emission tomography with 18 fluorodeoxyglucose in the staging and followup of lymphoma in the chest. Acta Oncol 1999; 38: 799-804.

52. Bangerter M, Moog F, Buchmann I, Kotzerke J, Griesshammer M, Hafner M, Elsner K, Frickhofen N, Reske SN, Bergmann L. Wholebody 2-[18F]-fluoro-2-deoxy-D-glucose positron emission tomography (FDG-PET) for accurate staging of Hodgkin's disease. Ann Oncol 1998; 9: 1117-22.

53. Jerusalem G, Warland V, Najjar F, Paulus $P$, Fassotte MF, Fillet G, Rigo P. Whole-body 18-FDG PET for the evaluation of patients with Hodgkin's disease and non-Hodgkin's lymphoma. Nucl Med Commun 1999; 20: 13-20.

54. Yap CS, Valk P, Aruannejad M, Seltzer MA, Phelps ME, Gambhir SS, Czernin J. FDG-PET influences the clinical management of lymphoma patients. J Nucl Med 2000, 41: $70 \mathrm{p}$.

55. Kostakoglu L, Leonard JP, Coleman M, Kuji I, Vallabhajosula S Goldsmith SJ. Comparison of FDG-PET and GA-67 SPECT in staging of lymphoma. J Nucl Med 2000; 41:118p.

56. Lin PC, Chu J, Pocock N. $18 F$ fluorodeoxyglucose imaging with coincidence dual-head gamma-camera (bybrid PET) for staging of lymphoma: comparison with Ga-67 scintigraphy. J Nucl Med 2000; 41:118p.

57. Wirth A, Seymour JF, Ware R, et al. Can F18 fluorodeoxyglucose positron emission tomography (PET) replace Gallium-67 scanning for staging Hodgkin's Disease and non-Hodgkin's lymphoma? Programs/ proceedings from the 36th annual meeting of the American Society of Clinical Oncology (www.conference-cast.com/asco/ vm2000)- NewOrleans LA, May 20-23, 2000.

58. Moog F, Bangerter M, Diederichs CG, Guhlmann A, Merkle E, Frichkhofen N, Reske SN. Extranodal malignant lymphoma: detection with FDG PET versus CT. Radiology 1998; 206: 475-81.

59. Moog F, Kotzerke J, Reske SN. FDG PET can replace bone scintigraphy inprimary staging of malignant lymphoma. J Nucl Med 1999; 40: 1407-13.

60. Carr R, Barrington SF, Madan B, O'Doherty MJ, Sauders CAB, VanderWalt J, Timothy AR. Detection of lymphoma in bone marrow by whole-body positron emission tomography. Blood 1988; 91: 3340-6.

61. Hoffman M, Kletter K, Diemling M, Becherer A, Pfeffel F, Petkov V, Chott A, Raderer M. Positron emission tomography with fluorine18-2-fluoro-2-deoxy-d-glucose (F18-FDG) does not visualize extranodal B-cell lymphoma of the mucosa associated lumphoid tissue (MALT)-type. Ann Oncol 1999; 10:1185-9.

62. Dewit M, Bumman D, Beyer W, Herbst K, Clausen M, Hossfeld DK. Whole-body positron emission tomography (PET) for diagnosis of residual mass in patients with lymphoma. Ann Oncol 1997; 8: S57-60.

63. Bangerter M, Moog F, Griesshammer M, Reske SN, Bergmann L. Role of whole-body FDG-PET in predicting relapse in residual masses after treatment of lymphoma. Br J Haematol 1998; 102: 148.

64. Mikhaeel NG, Timothy AR, Hain SF, ODoherty MJ. 18-FDG-PETfor the assessment of residual masses on CT following treatment of lymphomas. Ann Oncol 2000; 11: 14750.

65. Spaepen K, Stroobants S, Dupont P, van Steenweghen S, Thomas J, Peeters C, Verhoef G, Mortelmans L. Positron emission tomography with Fluorodeoxyglucose after first line chemotherapy in non-Hodgkin's lymphoma. J Nucl Med 2000; 41:70p.

66. Jerusalem G, Beguin Y, Fassotte MF, Najjar F, Paulus P, Rigo P, Fillet G. Whole-body positron emission tomography using $18 F$ fluorodeoxyglucose for posttreatment evaluation in Hodgkin's disease and non- 
Hodgkin's lymphoma has higher diagnostic and prognostic value than classical computed tomography scan imaging. Blood 1999; 94: 429-33.

67. Dimitrakopoulou-Strausss A, Strauss LG, Goldschimdt M, Lorenz WJ, Maier-Borst W, VanKaick G. Evaluation of tumor metabolism and multidrug resistance in patients with treated malignant lymphomas. Eur J Nucl Med 1995; 22: 434-42.

68. Weinblatt ME, Zanzi I, Belakhlef A, Babchyck B, Kochen J. False positive FDG-PET imaging of the thymus of a child with Hodgkin's disease. J Nucl Med 1997; 38: 888.

69. Sehweil AM, McKillop JH, Milroy R, Sayed MA, Ziada G, Banham W, Davidson KG, Ragib A, Omar YT, Abdel-Dayem HM. 201$T l$ scintigraphy in the staging of lung cancer, brest cancer and lymphoma. Nucl Med Commun 1990; 11: 263-9.

70. Mansberg R, Wadhwa SS, Mansberg V. Tl201 and Ga-67 scintigraphy in non-Hodgkin lymphoma. Clin Nucl Med 1999; 24: 239-42.

71. Roach PJ, Janicek MJ, Kaplan WD. Bone lymphoma: comparison of Tl-201 and Ga67 citrate scintigraphy in assessment of treatment response. Clin Nucl Med 1996; 21: 689-94.

72.Ivancevicz $V$, Nauck $C$, Worman B. Somatostatin receptor scintigraphy (SRS) with 111-indium-pentetreotide in Hodgkin (HL) and non-Hodgkin lymphomas (NHL) (abstract). EurJ Nucl Med 1994; 21: 726.

73. Lipp RW, Silly H, Ranner G, Dobnig H, Passath A, Leb G, Krejs GJ. Radiolabeled octreotide for the demonstration of somatostatin receptors in malignant lymphoma and lymphadenopathy. J Nucl Med 1995; 36: 13-8.

74. Lipp RW, Schnedl WJ, Aglas F, Ranner G, Beham Schmid C, Leb G, Sill H. Visualization of non-Hodgkin's lymphoma by bigh dosed somatostatin receptor specific scintigraphy and extended single photon emission tomography. Acta Med Austriaca 1999; 26: 105-8.

75. Van den Anker-Lugtemburg PJ, Lowenberg B, Lamberts SWJ, Oei HY, Valkema R, Krenning EP. Somatostatin receptor scintigraphy in the staging of non-Hodgkin lymphoma (abstract).
J Nucl Med 1996; 37:19P.

76. Van den Anker-Lugtemburg PJ, Krenning EP, Oei HY. Somatostatin receptor scintigraphy in the initial staging of Hodgkin's disease.

Br J Haematol 1996; 93: 96-103.

77. Reske SN. Recent advances in bone marrow scanning. Eur J Nucl Med 1991; 18: 203-21.

78. Altehoefer C, Blum U, Bathmann J, Wustenberg C, Uhrmeister P, Laubenberger J, Lange W, Schwazkopf J, Moser E, Langer M. Comparative diagnostic accuracy of magnetic resonance imaging and immunoscintigraphy for detection of bone marrow involvement in patients with malignant lymphomas. J Clin. Oncol 1997; 15: 1754-60.

79. Vandeberg BC, Lecouvert FE, Michaux L, Ferrant A, Maldague B, Malghem J. Magnetic resonance imaging of the bone marrow in hematological malignancies. Eur Radiol 1998; 8:1335-44.

80. Krause T, Eisenmann N, Reinhardt $\mathrm{M}$, Bathmann J, Altehoefer C, Finke J, Moser E. Bone marrow scintigraphy using technetium-99m antigranulocyte antibody in malignant lymphomas. Ann Oncol 1999; 10: 79-85.

81. Linden A, Zankovich R, Theissen P, Diehl $\mathrm{V}$, Schicha H. Malignant Lymphoma: bone marrow imaging versus biopsy. Radiology 1989; 173: 335-9.

82. Chatal JF, Mahé M. Therapeutic use of radiolabeled antibodies. in: Murray IPC, Ell PJ, eds. Nuclear Medicine in Clinical Diagnosis and Treatment (2ed). Edinburgh: Churchill Livingstone; 1998 p 1101.

83. Press OW. Radiolabeled antibody therapy of B-cell lymphomas. Semin Oncol 1999; 26 : 58-65.

84. Wiseman GA, White CA, Witzig TE, Gordon LI, Emmanouilides C, Raubitschek A, Janakiraman N, Gutheil J, Schilder RJ, Spies S, Silverman DH, Grillo-López AJ. Radioimmunotherapy of relapsed nonHodgkin's lymphoma with zevalin, a ${ }^{90} Y$ labeled anti-CD2O monoclonal antibody. Clin Cancer Res 1999, 5:3281-3286s.

Recebido: 02/04/01

Aceito: 08/06/01 